\title{
Observer-Based Fuzzy Control Scheme for a Class of Nonlinear Systems
}

\author{
Hugang $\operatorname{Han}^{*}$ Member
}

This paper addresses the fuzzy control problem for a class of nonlinear systems using the Lyapunov synthesis approach. In order to deal with cases where the system state is unavailable, a state observer is proposed. Consequently, the whole system behavior can be attributed to a kind of the standard singularly perturbed form. At the same time, to deal with the gap, if any, between the real state and its estimated value from the state observer, we view it as a part of system disturbance, and propose a unique way to deal with the disturbance, i.e., adopt a switching function with an alterable coefficient, which is tuned by adaptive law based on the tracking error between the output of the considered system and the desired value. Finally, it is shown that the fuzzy controller proposed guarantees the tracking error will shrink to zero, while maintaining all signals involved in the system stable.

Keywords: Stability, State observer, Lyapunov synthesis approach, Fuzzy approximator

\section{Introduction}

A large amount of research has focused on fuzzy control systems with a keyword of system stability since the early 1990's (e.g. ${ }^{(1)-(6)}$ and references therein). In such a fuzzy control system, the Lyapunov synthesis approach is used to construct a stable controller, and to deal with the uncertainties in the system, the traditional adaptive control theory is merged with fuzzy approximation theory ${ }^{(2)}$ where the unknown functions in the system are approximated by parameterized fuzzy approximators. In general, there are two basic tasks should be solved in the context of a stable nonlinear control system. One is how to deal with the unknown functions in the system to be controlled, and the other is how to observe the system state which usually is used in developing control system directly. For the first task, as mentioned previously, the fuzzy approximator surely is a very effective tool to be considered. Nevertheless there exists a reconstruction error between the exact function and its corresponding fuzzy approximator. To deal with such an error, the upper bound of the error is assumed to be known ${ }^{(7)(10)}$. In fact, this kind of the upper bounds about the uncertainties is not easy to determine in advance of designing the control system, therefore, to be safe for the system stability, a larger magnitude of assumption about the uncertainties is supposed, and consequently, a bigger chattering in control inputs is always involved. For the second task, active research has been carried out using the concept of the fuzzy state observer in fuzzy control systems ${ }^{(8)-(10)}$. However, their is not a clear clue that shows the connection between the real control state, observer, fuzzy approximator, and system controller.

In this paper, our goal is to design a fuzzy controller for

\footnotetext{
* Department of Management and Information Sciences, Hiroshima Prefectural University

562 Nanatsuka-cho, Shobara-shi, Hiroshima 727-0023, Japan
}

a class of nonlinear systems with disturbance, in which a state observer is proposed instead of using the unavailable system state. Considering the part closely related with the state observer as a subsystem, the whole system behavior, thus, becomes a kind of the standard singularly perturbed form ${ }^{(13)-(15)}$, in which the gap between the real state and its corresponding value from the state observer decays to order $O(\epsilon)^{\dagger}$ at a very fast speed by choosing an arbitrarily small constant $\epsilon$. Also until then, the gap is treated as part of system disturbance. To deal with the reconstruction error regarding the fuzzy approximator, we adopt a saturation function with an alterable coefficient, which is tuned by an adaptive law based on the tracking error, in stead of the upper bound assumptions as well as treating the disturbance. The adaptive laws to adjust all parameters will be developed based on the Lyapunov synthesis approach. It is shown that the proposed fuzzy controller guarantees the tracking error, between the output of the considered system and the desired value, to be shrunken to zero, while maintaining all signals involved in the system stable. The remainder of this paper is arranged as follows. Section 2 describes a class of nonlinear systems with disturbance. In section 3, first we give a brief review of the fuzzy model so that we can view it as an approximator to deal with the unknown functions in the system, afterwards a state observer is presented, then we exploit the fuzzy approximator to develop a fuzzy control system with the state observer, for which we also give a detailed analysis of its stability. Also, an illustration example in sction 4 will be shown in order to confirm our control scheme. Finally, the conclusion is given in section 5 of this paper.

\footnotetext{
${ }^{\dagger}$ A vector function $f(t, \epsilon) \in \mathcal{R}^{n}$ is said to be $O(\epsilon)$ over an interval $\left[t_{1}, t_{2}\right]$ if there exist positive constants $k$ and $\epsilon^{*}$ such that$$
\|f(t, \epsilon)\| \leq k \epsilon, \quad \epsilon \in\left[0, \epsilon^{*}\right], \quad t \in\left[t_{1}, t_{2}\right]
$$$$
\text { where }\|\cdot\| \text { is the Euclidean norm }{ }^{(16)} \text {. }
$$ 


\section{Problem Statement}

This paper focuses on the design of fuzzy control algorithms for a class of nonlinear systems whose equation of motion can be expressed in the canonical form:

$$
\left\{\begin{array}{l}
x^{(n)}=f(X)+b(X) u+d \\
y=x
\end{array}\right.
$$

where $X^{T}=\left[x, \dot{x}, \ldots, x^{(n-1)}\right]$ is the system state; $u$ is the control input; $y$ is the output; $f$ is a smoothly unknown nonlinear function; $b$ is the control gain; $d$ denotes an unknownly bounded disturbance in the system. It should be noted that more general classes of nonlinear control problems can be transformed into this structure $^{(3)}$. Also, system (1) can be rewritten in state-space representation,

$$
\left\{\begin{array}{l}
\dot{X}=A X+B[f(X)+b(X) u+d] \\
y=C^{T} X
\end{array}\right.
$$

where,

$$
\begin{aligned}
& A=\left[\begin{array}{ccccccc}
0 & 1 & 0 & 0 & \cdots & 0 & 0 \\
0 & 0 & 1 & 0 & \cdots & 0 & 0 \\
\vdots & \vdots & \vdots & \vdots & \ddots & \vdots & \vdots \\
0 & 0 & 0 & 0 & \cdots & 0 & 1 \\
0 & 0 & 0 & 0 & \cdots & 0 & 0
\end{array}\right] \\
& B^{T}=\left[\begin{array}{lllll}
0 & 0 & \ldots & 0 & 1
\end{array}\right] \\
& C^{T}=\left[\begin{array}{lllll}
1 & 0 & \ldots & 0 & 0
\end{array}\right]
\end{aligned}
$$

and $X^{T}=\left[x_{1}, x_{2}, \ldots, x_{n}\right]=\left[x, \dot{x}, \ldots, x^{(n-1)}\right]$ where not all $x_{i}$ are assumed to be available for measurement in this paper. In order to pay attention to a clearer control scheme, we suppose that the control gain $b=1$ throughout this paper.

The control objective is to design a fuzzy controller so that the overall system is stabilized and the output $x$ is forced to follow a desired value $x_{d}$. Defining the tracking error,

$$
\tilde{x}=x-x_{d}
$$

the problem is thus to design a controller $u$ which ensures that $\tilde{x} \rightarrow 0$, as $t \rightarrow \infty$. The nonlinear function $f$ in (2) is unknown, so before developing our control algorithm we have to solve the problem of approximating $f$. In the following section, it will be shown that using fuzzy IF-THEN rules, the unknown function $f$ can be approximated by a fuzzy approximator.

\section{Fuzzy Control Scheme}

3.1 Fuzzy Approximator The fuzzy model addresses the imprecision of the input and output variables directly by defining them with fuzzy sets in the form of membership functions. The basic configuration of the fuzzy model includes a fuzzy rules base, which consists of a collection of IF-THEN fuzzy rules in the following form:

Rule j: IF $z_{1}$ is $A_{j}^{1}, \ldots, z_{n}$ is $A_{j}^{n}$,

THEN $f$ is $w_{j}$ where $A_{j}^{i}$ is a fuzzy set, $z_{i}$ is a measurable variable in the antecedent of a fuzzy rule, $f$ is the output variable in the consequent of a fuzzy rule, and $w_{j}$ is a fuzzy set or singleton value. Now, we consider a fuzzy model with a singleton consequent, product inference, Gaussian membership function in the antecedent, and central average defuzzifier, hence, such a fuzzy model can be written as

$$
\mathcal{F}(Z)=W^{T} \cdot G(Z)
$$

where $Z^{T}=\left[z_{1}, z_{2}, \ldots, z_{n}\right], W^{T}=\left[w_{1}, w_{2}, \ldots, w_{N}\right]$ with $N$ being the number of fuzzy rules; $G^{T}(Z)=$ $\left[g_{1}(Z), g_{2}(Z), \ldots, g_{N}(Z)\right]$ with $g_{j}(Z)=\frac{\prod_{i=1}^{n} \mu_{A_{j}^{i}}\left(z_{i}\right)}{\sum_{j=1}^{N} \prod_{i=1}^{n} \mu_{A_{j}^{i}}\left(z_{i}\right)}$ where $\mu_{A_{j}^{i}}\left(z_{i}\right)$ is a Gaussian membership function, defined by

$$
\mu_{A_{j}^{i}}\left(z_{i}\right)=\exp \left[-\left(\frac{z_{i}-\xi_{j}^{i}}{\sigma_{j}^{i}}\right)^{2}\right] \ldots \ldots \ldots \ldots \ldots
$$

where $\xi_{j}^{i}$ indicates the position, and $\sigma_{j}^{i}$ indicates the variance of the membership function.

We now can show an important property of the fuzzy system above. As shown by Wang et $a l^{(9)}$, the fuzzy system has the same pattern as a neural network. Exactly as a neural network, which has powerful abilities of learning and approximation, a fuzzy system with Gaussian membership is capable of uniformly approximating any well-defined nonlinear function over a compact set to any degree of accuracy ${ }^{(17)}$. In this paper, we use terms fuzzy universal approximator or fuzzy approximator to refer to the fuzzy system. Since the fuzzy universal approximator is characterized by parameter vectors $W$, the fuzzy approximator, $\mathcal{F}^{*}=W^{* T} \cdot G(Z)$, does contain an optimal vector $W^{*}$.

3.2 State Observer To deal with the unknown functions such as $f(X)$, we will employ the fuzzy approximator above to estimate them. In the fuzzy approximator, as the input variables the system state is often used. However, as mentioned previously, not all $x_{i}$ are assumed to be available for measurement in this paper, therefore, first of all we have to design a state observer. We estimate the state $x_{i}$ using the observer ${ }^{(9)}$ (10) (15)

$$
\left\{\begin{array}{l}
\dot{\hat{x}}_{i}=\hat{x}_{i+1}+\frac{\alpha_{i}}{\epsilon^{i}}\left(y-\hat{x}_{1}\right), \quad i=1,2, \ldots, n-1 \\
\dot{\hat{x}}_{n}=\frac{\alpha_{n}}{\epsilon^{n}}\left(y-\hat{x}_{1}\right) s
\end{array}\right.
$$

where $\epsilon$ is a positive parameter to be specified. The positive constant $\alpha_{i}$ is chosen such that the roots of

$$
s^{n}+\alpha_{1} s^{n-1}+\cdots+\alpha_{n-1} s+\alpha_{n}=0 \cdots \cdots \cdots
$$

have negative real parts. Like (1), the state observer (6) can be rewritten in state-space representation,

$$
\dot{\hat{X}}=A \hat{X}+D(\epsilon) L C^{T}(X-\hat{X}) \cdots \cdots \cdots \cdots
$$

where,

$$
D(\epsilon)=\left[\begin{array}{cccc}
\frac{1}{\epsilon} & & & \\
& \frac{1}{\epsilon^{2}} & & \\
& & \ddots & \\
& & & \frac{1}{\epsilon^{n}}
\end{array}\right],
$$




$$
\begin{aligned}
& L^{T}=\left[\alpha_{1}, \alpha_{2}, \ldots, \alpha_{n}\right], \\
& \hat{X}^{T}=\left[\hat{x}_{1}, \hat{x}_{2}, \ldots, \hat{x}_{n}\right]=\left[\hat{x}, \hat{\dot{x}}, \ldots, \hat{x}^{(n-1)}\right] .
\end{aligned}
$$

Now, we define a matrix $N(\epsilon)$ as follows.

$$
N(\epsilon)=\left[\begin{array}{cccc}
\epsilon^{n-1} & & & \\
& \epsilon^{n-2} & & \\
& & \ddots & \\
& & & \epsilon^{0}
\end{array}\right] .
$$

Using the special structure of the matrices $N(\epsilon), D(\epsilon)$, $B, C$, and $L$, it can be shown that

$$
\left\{\begin{array}{l}
N^{-1}(\epsilon) B=B \\
N^{-1}(\epsilon)\left[A-D(\epsilon) L C^{T}\right] N(\epsilon)=\frac{1}{\epsilon}\left[A-L C^{T}\right]
\end{array}\right.
$$

Let

$$
e_{i}=\frac{1}{\epsilon^{n-i}}\left(x_{i}-\hat{x}_{i}\right)
$$

and $E^{T}=\left[e_{1}, e_{2}, \ldots, e_{n}\right]=\left[e, \dot{e}, \ldots, e^{n-1}\right]$, which is the scaling estimation error. Thus, we have

$$
E=N^{-1}(\epsilon)(X-\hat{X})
$$

From (2), (8), (9), and (11), it follows that

$$
\dot{E}=\frac{1}{\epsilon}\left(A-L C^{T}\right) E+B(f(X)+B u+d)
$$

where the characteristic equation of matrix $\left(A-L C^{T}\right)$ is (7). Including the state observer, at this stage the whole system behavior is dominated by (2), and (12). This is the standard singularly perturbed form. If attention is paid to term $\frac{1}{\epsilon}$ in (12), we can easily see that the evolutions of $X$, and $E$ do have an absolutely different transient speed, in which (12) is called the fast model whereas (2) is called the slow model ${ }^{(14)}$. In the following subsection, we will take advantage of the evolutions' different transient speeds to develop the fuzzy control system.

3.3 Structure of Controller In this paper, we adopt the variable structure theory to construct our adaptive fuzzy control system. The sliding mode hyperplane is firstly defined as

$$
s=\left(\frac{d}{d t}+\lambda\right)^{n-1} \tilde{x} \quad \text { with } \lambda>0
$$

where $\lambda$ defines the bandwidth of the error dynamics of the system. The equation defines a time-varying hyperplane in $\mathcal{R}^{n}$ on which the tracking error $\tilde{x}$ decays exponentially to zero, so that perfect tracking can be asymptotically obtained by maintaining this condition. In this case the control objective becomes the design of a controller that ensures $s=0$. The time derivative of the error metric can be written as

$$
\dot{s}=u+d+f(X)-x_{d}^{(n)}+\Lambda^{T} \tilde{X}
$$

where $\Lambda^{T}=\left[0, \lambda^{n-1},(n-1) \lambda^{n-2}, \ldots,(n-1) \lambda\right], \tilde{X}=$ $X-X_{d}, X_{d}^{T}=\left[x_{d}, \dot{x}_{d}, \ldots, x_{d}^{(n-1)}\right]$. Referring to system (1), it naturally suggests that when $f(X)$ and the boundedness of the disturbance $d$ are known, a controller of the form

$$
u=-k_{d} s+a_{r}-f(X)-d^{*} \cdot \operatorname{sgn}(s) .
$$

where $k_{d}>0,|d| \leq d^{*}, a_{r}=x_{d}^{(n)}-\Lambda^{T} \tilde{X}$. Substituting (15) into (14), we have

$$
\dot{s}=-k_{d} s+d-d^{*} \operatorname{sgn}(s)
$$

where, $d^{*}$ is the upper bound of the disturbance $d$, i.e., $|d| \leq d^{*}, \operatorname{sgn}(s)$ is a switching function, i.e.,

$$
\operatorname{sgn}(s)=\left\{\begin{aligned}
1, & \text { if } s \geq 0 \\
-1, & \text { if } s<0
\end{aligned}\right.
$$

which has a property that $\operatorname{sgn}(\mathrm{s}) \cdot s=|s|$. Hence,

$$
\begin{aligned}
\dot{s} s & =-k_{d} s^{2}+d s-d^{*}|s| \\
& \leq-k_{d} s^{2} \ldots \ldots \ldots
\end{aligned}
$$

which leads to $s \rightarrow 0$, consequently $\tilde{x} \rightarrow 0$ as $t \rightarrow \infty$. However, the problem is how $u$ can be determined when $f(X)$ as well as the upper bound $d^{*}$ for $d$, is unknown. What is more, the state $X$ and the sliding mode $s$ involving $X$ can not be used in the controller directly due to the problem with $X$ 's measurement.

Using the estimated state $\hat{X}$ instead of $X$, we redefine the sliding mode hyperplane

$$
\hat{s}=\left(\frac{d}{d t}+\lambda\right)^{n-1}\left(\hat{x}-x_{d}\right)
$$

Using the relation $\hat{x}=x-\epsilon^{n-1} e$ from (11), $\hat{s}$ can be rewritten as

$$
\hat{s}=s-\epsilon^{n-1} \Lambda_{1}^{T} E
$$

where $\Lambda_{1}^{T}=\left[\lambda^{n-1},(n-1) \lambda^{n-2}, \ldots,(n-1) \lambda, 1\right]$. Taking the time derivative of both sides of (20) it follows that

$$
\begin{aligned}
\dot{\hat{s}}= & f(X)+u+\Lambda^{T}\left(\hat{X}-X_{d}\right)-x_{d}^{(n)} \\
& +d+\Lambda^{T}\left(N(\epsilon)-\epsilon^{n-1} I\right) E \\
& -\epsilon^{n-1}\left(x^{(n)}-\hat{x}^{(n)}\right) \ldots \ldots \ldots \ldots \ldots \ldots \ldots \ldots
\end{aligned}
$$

Let us focus our attention on the last term above. Since the state observer as shown in (6) does not need the estimated value of $x^{(n)}$ in the system, the gap, if any, between $x^{(n)}$ and $\hat{x}^{(n)}$ does not affect the system at all, and it is reasonable to suppose that $x^{(n)}-\hat{x}^{(n)}=0$. Thus, (21) becomes,

$$
\dot{\hat{s}}=f(X)+u-\hat{a}_{r}+d_{e}
$$

where $\hat{a}_{r}=x_{d}^{(n)}-\Lambda^{T}\left(\hat{X}-X_{d}\right)$, and

$$
d_{e}=d+\Lambda^{T}\left(N(\epsilon)-\epsilon^{n-1} I\right) E .
$$

As shown later on, the scaling estimation error $E$ is bounded. Considering the boundedness of $E$, and $d$, 
the inequality

$$
\left|d_{e}\right| \leq d_{e}^{*}
$$

is satisfied for some $d_{e}^{*}$. We should note that the value of $d_{e}^{*}$ does not involve in the system design, therefore, there is no need to know the value.

To proceed with the system development, one task left is how to deal with the unknown function $f(X)$. Here in this paper we employ the fuzzy approximator described previously to estimate it using the estimated state $\hat{X}$. Let us denote $f^{*}(\hat{X})=W_{f}^{* T} G_{f}(\hat{X})$ to be the optimal fuzzy approximator of the unknown function $f(X)$. There is a small positive value $\varepsilon_{f}^{*}$ such that the error,

$$
\varepsilon_{f}=f(X)-f^{*}(\hat{X})
$$

which is referred to as reconstruction error, satisfies the following inequality ${ }^{(17)}$,

$$
\left|\varepsilon_{f}\right| \leq \varepsilon_{f}^{*}
$$

We also should note that the value of $\varepsilon_{f}^{*}$ does not need to be known in this paper.

Apparently, the optimal vector $W_{f}^{*}$ in the optimal fuzzy approximator is unknown either, so its estimate, denoted $\hat{f}(\hat{X})=\hat{W}_{f}^{T} G_{f}(\hat{X})$ is adopted. Inspired by the control structure in (15), our fuzzy controller is now described as

$$
u=u_{f d}+u_{f z}+u_{s d}
$$

where $u_{f d}, u_{f z}$, and $u_{s d}$ are an error's feedback component, fuzzy component and sliding component, respectively. In the concrete, the error's feedback component $u_{f d}$, expressed by,

$$
u_{f d}=-k_{d} \hat{s}_{\phi}+\hat{a}_{r}, \quad k_{d}>0
$$

is a kind of feedback of tracking error $\tilde{x}$, where

$$
\begin{gathered}
s_{\phi}=\hat{s}-\phi \cdot \operatorname{sat} \frac{\hat{s}}{\phi} \ldots \ldots \ldots \ldots \ldots \ldots \ldots \ldots \ldots \ldots \\
\operatorname{sat}\left(\frac{\hat{s}}{\phi}\right)=\left\{\begin{array}{cl}
1, & \frac{\hat{s}}{\phi} \geq 1 \\
-1, & \frac{\hat{s}}{\phi} \leq 1 \\
\frac{\hat{s}}{\phi}, & \text { otherwise }
\end{array}\right.
\end{gathered}
$$

$\phi$ is a small constant to be specified. The fuzzy component $u_{f z}$, expressed by,

$$
u_{f z}=-\hat{f}(\hat{X})-\hat{\varepsilon}_{f} \cdot \operatorname{sat}\left(\frac{\hat{s}}{\phi}\right)
$$

will cover the unknown function $f(X)$, and attempt to compensate the estimating error. At the same time, the adaptive laws are synthesized by

$$
\begin{aligned}
\dot{\hat{W}}_{f} & =\Gamma_{f} G_{f}(\hat{X}) \hat{s}_{\phi} \ldots \ldots \ldots \ldots \ldots \ldots \ldots \ldots \ldots \ldots \ldots \ldots \ldots \ldots \ldots \ldots \ldots
\end{aligned}
$$

where $\hat{W}_{f}$, and $\hat{\varepsilon}_{f}$ are the estimates of $W_{f}^{*}$, and $\varepsilon_{f}^{*}$, respectively; $\Gamma_{f}$, and $\gamma_{f}$ are an appropriate symmetric positive definite matrix and a positive constant, respectively, which determine the rates of adaptation.
The sliding component $u_{s d}$, expressed by,

$$
u_{s d}=-\hat{d}_{e} \cdot \operatorname{sat}\left(\frac{\hat{s}}{\phi}\right)
$$

copes with the disturbance $d$ in (1) and the scaling estimation error $E$ in (11). And the coefficient $\hat{d}_{e}$, which is the estimate of $d_{e}^{*}$, is tuned by an adaptive law as follows:

$$
\dot{\hat{d}}_{e}=\gamma_{d_{e}}\left|\hat{s}_{\phi}\right|
$$

where $\gamma_{d_{e}}$ is the rate of adaptation as well.

Regarding the adaptive fuzzy controller above, we give the following remarks.

Remark 1 In a general way, to cancel the influence of the uncertainty $d$ on the control system, a switching function like $d_{e}^{*} \operatorname{sgn}(\hat{s})$ is used. In this case, the value of the upper bound $d_{e}^{*}$ in (24) should be known. Actually, in a control system, such a upper boundary value is difficult to exactly be known, and a larger boundary value is adopted to guarantee the stability of the control system. However, in the end, a bigger chattering problem occurs. In our way, it does not need the upper boundary value, and the coefficient is tuned by an adaptive law based on the tracking error.

Remark 2 In the fuzzy component (31), the last term on the right actually reflects the component for compensation of the approximating error, $(f(X)-\hat{f}(\hat{X}))$. If it has a better approximateness, i.e., the approximating error is smaller, then the last term on the right of (31) is also smaller. However, if the approximating error is larger, then it automatically also becomes larger, ensuring the stability of the system.

Remark 3 In (15), when the tracking error $\tilde{x}$ enters around the sliding surface, sign function $\operatorname{sgn}(s)$ begins working frequently so that such a control law leads to control chattering. Chattering is undesirable in practice because it involves high control activity, and further may excite unmodeled high frequency plant dynamics. This problem can be eliminated by adopting a saturation function $\operatorname{sat}(\hat{s} / \phi)(30)$ instead of $\operatorname{sgn}(\hat{s})$, and a smoothed sliding mode $s_{\phi}(29)$ instead of $s$.

3.4 Analysis of stability Now, consider the following Lyapunov function candidate,

$$
V_{1}=\frac{1}{2}\left(\hat{s}_{\phi}^{2}+\frac{1}{\gamma_{d_{e}}} \tilde{d}_{e}^{2}+\tilde{W}_{f}^{T} \Gamma_{f}^{-1} \tilde{W}_{f}+\frac{1}{\gamma_{f}} \tilde{\varepsilon}_{f}^{2}\right)
$$

where,

$$
\begin{aligned}
\tilde{d}_{e} & =d_{e}^{*}-\hat{d}_{e} \cdots \\
\tilde{W}_{f} & =W_{f}^{*}-\hat{W}_{f} \\
\tilde{\varepsilon}_{f} & =\varepsilon_{f}^{*}-\hat{\varepsilon}_{f} \cdots
\end{aligned}
$$

From (22), (27-28), (31), and (34), we have

$$
\begin{aligned}
\hat{s}_{\phi} \dot{\hat{s}}_{\phi}= & \left(\varepsilon_{f}+f^{*}(\hat{X})-\hat{f}(\hat{X})\right) s_{\phi}+d_{e} \hat{s}_{\phi}-k_{d} \hat{s}_{\phi}^{2} \\
& -\hat{\varepsilon}_{f}\left|s_{\phi}\right|-\hat{d}_{e}\left|s_{\phi}\right| \cdots \ldots \ldots \ldots \ldots \ldots \ldots \ldots
\end{aligned}
$$


where the relation, $\varepsilon_{f}=f(X)-f^{*}(\hat{X})$, and the fact that $\hat{s}_{\phi} \cdot \operatorname{sat}(\hat{s} / \phi)=\left|\hat{s}_{\phi}\right|$ have been used.

Taking the derivative of the Lyapunov function yields,

$$
\dot{V}_{1}=\hat{s}_{\phi} \dot{\hat{s}}_{\phi}-\frac{1}{\gamma_{d_{e}}} \tilde{d}_{e} \dot{\hat{d}}_{e}-\tilde{W}_{f}^{T} \Gamma_{f}^{-1} \dot{\hat{W}}_{f}-\frac{1}{\gamma_{f}} \tilde{\varepsilon}_{f} \dot{\hat{\varepsilon}}_{f}
$$

Substituting (40), adaptive laws (32-33), and (35) into (41), it follows that

$$
\begin{aligned}
& \dot{V}_{1}=\left(\varepsilon_{f}+f^{*}(\hat{X})-\hat{f}(\hat{X})\right) s_{\phi} \\
& +d_{e} \hat{s}_{\phi}-k_{d} \hat{s}_{\phi}^{2}-\hat{\varepsilon}_{f}\left|s_{\phi}\right|-\hat{d}_{e}\left|s_{\phi}\right| \\
& -\left(d_{e}^{*}-\hat{d}_{e}\right)\left|\hat{s}_{\phi}\right| \\
& -\left(W_{f}^{*}-\hat{W}_{f}\right) G_{f}(\hat{X}) \hat{s}_{\phi} \\
& -\left(\varepsilon_{f}^{*}-\hat{\varepsilon}_{f}\right)\left|\hat{s}_{\phi}\right| \\
& =-k_{d} \hat{s}_{\phi}^{2}+\varepsilon_{f} \hat{s}_{\phi}-\varepsilon_{f}^{*}\left|s_{\phi}\right| \\
& +d_{e} \hat{s}_{\phi}-d_{e}^{*}\left|\hat{s}_{\phi}\right| \\
& <-k_{d} \hat{s}_{\phi}^{2}
\end{aligned}
$$

where the facts that $\left|\varepsilon_{f}\right|<\varepsilon_{f}^{*},\left|d_{e}\right| \leq d_{e}^{*}$ have been used. Therefore, all signals in (36), which also are signals involved in the system, are bounded, and $\hat{s}_{\phi} \rightarrow 0$, furthermore, $\hat{s} \rightarrow 0$ as $t \rightarrow \infty$, which also means $\left(\hat{x}-x_{d}\right) \rightarrow 0$, as $t \rightarrow \infty$.

However, our goal is to drive, not the estimated state $\hat{x}$, but the real state $x$ to follow the desired value. To this end, one way, the direct way, is to show $x$ surely is following $x_{d}$, and another way, the indirect way, is to show that $\hat{x}$ is equal to $x$ so that the error between them decays to almost zero at a very fast speed. Here, we take the indirect approach. Let us pay attention to the fast model (12). Consider the following Lyapunov function candidate,

$$
V_{2}=E^{T} P E
$$

where $P^{T}=P>0$ is the solution of the Lyapunov equation,

$$
P\left(A-L C^{T}\right)+\left(A-L C^{T}\right)^{T} P=-I
$$

Based on the analysis, the boundedness of controller (27) is guaranteed. Therefore, inequality

$$
|f(X)+b(X) u+d| \leq k
$$

is satisfied for some $k \geq 0$ subject to Assumption 1, 2. Substituting (44) into the time derivative of $V_{2}$, it follows that

$$
\begin{aligned}
\dot{V}_{2} & \leq-\frac{1}{\epsilon}\|E\|^{2}+2 k\|P B\|\|E\| \\
& \leq-\frac{\gamma}{\epsilon} V_{2}, \quad \text { if } V_{2} \geq \epsilon^{2} \beta \ldots
\end{aligned}
$$

where $\gamma=\frac{1}{2 \lambda_{\max }(P)}$, and $\beta=16\|P B\|^{2} k^{2} \lambda_{\max }(P)$. This implies,

$$
V_{2} \leq V_{2}(0) e^{-\frac{\gamma}{\epsilon} t}
$$

We can get that there exists

$$
\mathcal{T}=\frac{\epsilon}{\gamma} \ln \left(\frac{V_{2}(0)}{\epsilon^{2} \beta}\right) .
$$

such that for $t \geq \mathcal{T}, V_{2}$ satisfies

$$
V_{2} \leq \epsilon^{2} \beta
$$

From (43), we have $\|E\|^{2} \leq \frac{1}{\lambda_{\min }(P)} V_{2}$. Therefore, it follows that

$$
\|E\| \leq \mu \epsilon, \quad \text { if } t \geq \mathcal{T}
$$

where $\mu=\sqrt{\frac{\beta}{\lambda_{\min }(P)}}$. Therefore, the scaling estimation error decays to the order $O(\epsilon)$. Since $\epsilon$ can be chosen arbitrarily small, we can make $\mathcal{T}$ in (48) arbitrarily small as well. Consequently, considering the relation in (11), we conclude the estimated state $\hat{X}$ closely follows its real state $X$ at a very fast speed, i.e., $\hat{X} \rightarrow X$. Furthermore, we have $x \rightarrow x_{d}$.

The stability of the closed-loop system described by (1), (6), and (27-35) is thus estabilished in the following theorem.

Theorem 1 If the plant (1) is controlled by (27-30), (31), and (34) with the adaptive laws (32-33), (35), and the state observer (6), then all signals involved in the control system will remain bounded, and the tracking error will asymptotically shrink to zero; at the same time that the estimated state follows its real state.

\section{Simulation Example}

To clarify the proposed design procedure, we apply the design scheme developed in the previous section to control a nonlinear system which is described as

$$
\dot{x}=\frac{1-e^{-x}}{1+e^{-x}}+u+d
$$

where $d$, as shown in Fig.3 (dashed line), is the distrubance in the form of

$$
d= \begin{cases}1.5 & 2.0 \leq t \leq 2.5 \\ -1.5 & 3.5 \leq t \leq 4.0 \\ 0 & \text { otherwise }\end{cases}
$$

Without the control, i.e., $u=0$, it can be easily seen that the system is unstable, because of $f=\frac{1-e^{-x}}{1+e^{-x}}>0$ for $x>0$, and $f=\frac{1-e^{-x}}{1+e^{-x}}<0$ for $x<0$ (Fig.1). The control objective is to force the system state $x$ to the

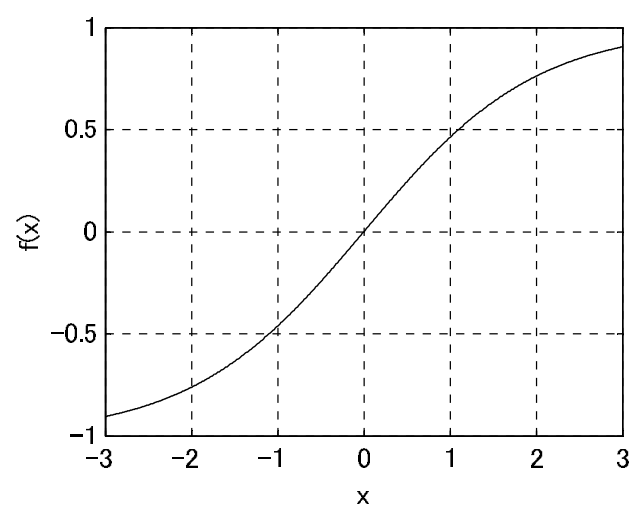

Fig. 1. Nonlinear function $f$ in 51 


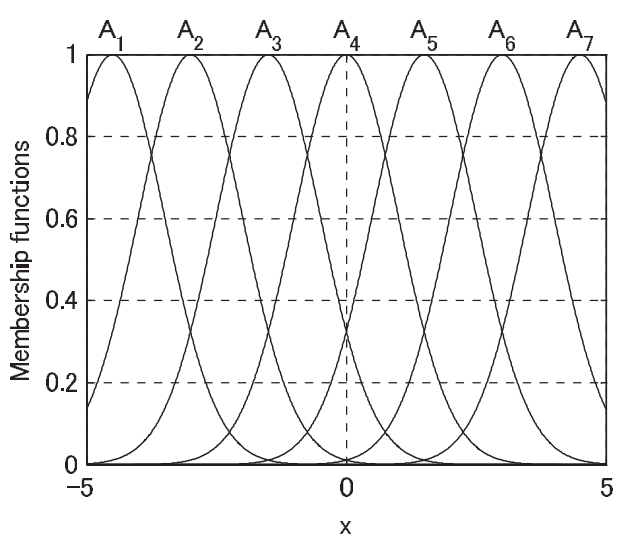

Fig. 2. Membership functions in precedent

origin, i.e., $x_{d}=0$. The simulation is conducted with the following fuzzy rules:

$$
R_{j}: \text { IF } x \text { is } A_{j} \text { THEN } f \text { is } w_{j}
$$

where $j$ is a rule's number; $A_{j}$ is a fuzzy set, and $w_{j}$ is a singleton value. So, first of all, the problem is how to divide the domain of $x$ into a few fuzzy sets. As shown in Fig.4, for such a curve of function $f$, it is reasonable to consider five to seven fuzzy sets over the domain by our experience (or something akin to intuition). The following reason strongly supports our consideration. In most cases, such an unknown function is approximately described in the form of fuzzy rules, which is exactly the form of a human-language-like description, through the expert's knowledge, experience and so on. This is the primary reason we use fuzzy control; otherwise we could use the other soft computing methods such as a neural network, genetic algorithm, or maybe a purely mathematical method in the sense of the approximating function. So in the case of describing $f$ by an (human) expert, how many cases should be considered? Five to seven seems reasonable. In this simulation, we adopt the number seven, in other words, there are seven fuzzy sets over the domain of $x$. Consequently, it yields seven fuzzy rules at most, where each fuzzy rule $R_{j}(j=1,2, \cdots, 7)$ has a consequent $w_{j}$. Furthermore, seven fuzzy sets are given as in Fig.2, and $w_{j}$ is initially assigned 1 and to be tuned by adaptive laws.

Control law (27) was used. The error's feedback component $u_{f d}$ is synthesized by (28) where $k_{d}=1, \gamma_{d_{g}}=$ 0.2 , and $\phi=0.01$. The fuzzy component $u_{f z}$ is synthesized by (31), (32), and (33) where $\Gamma_{f}=0.1 I$ with $I$ being an appropriate identity matrix, $\gamma_{f}=0.2$. Further more, the sliding component is determined by (34), and (35) where $\gamma_{d_{e}}=0.2$. In addition, we take the values that $\lambda=\alpha_{1}=1, \varepsilon=0.01$, and initial state $x(0)=3$.

Simulation results are shown in Figs.3-5. Fig.3 (solid line) shows the evolution of $x$ where a good performance is observed even over the time periods of $2.0 \leq t \leq 2.5$, and $3.5 \leq t \leq 4$ during which the disturbance $d$ occurred. The amount of control effort required to achieve the above level of performance is illustrated in Fig.4, in which we can see that $u$ was promptly corresponding to the disturbance. The error between the estimated state $\hat{x}$ from the state observer and its real state $x$ is shown in

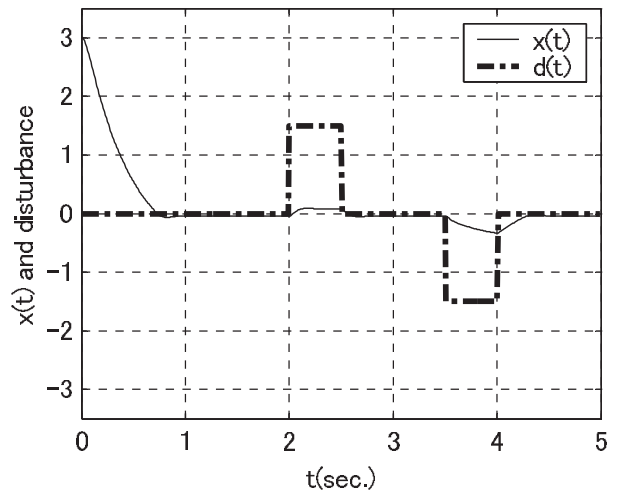

Fig. 3. Evolution of state $\mathrm{x}$

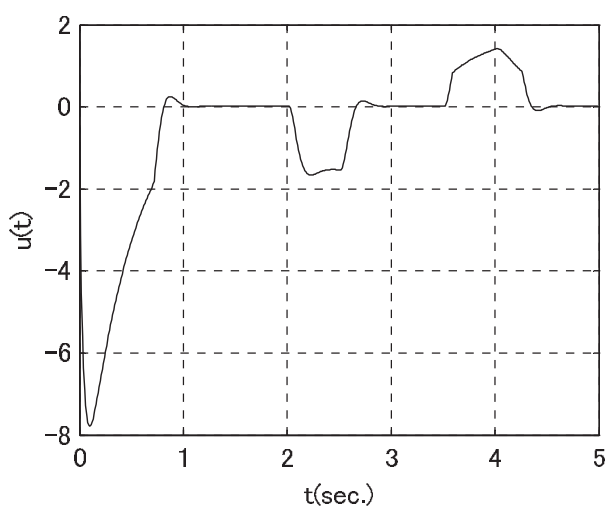

Fig. 4. Amount of control law $u$

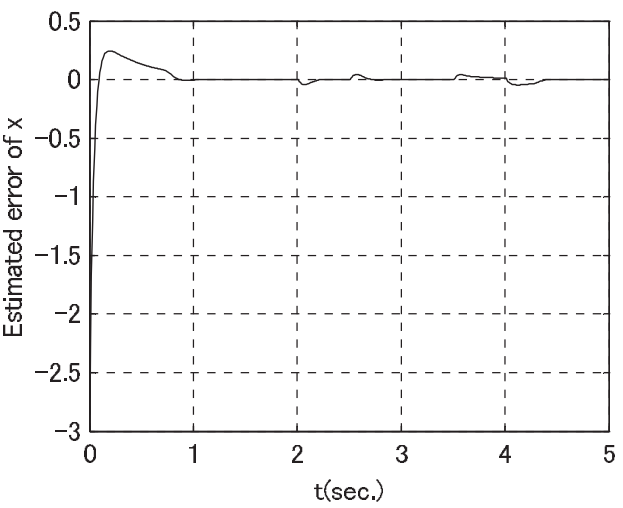

Fig. 5. Estimated error $(\hat{x}-x)$.

Fig.5 which exhibits clearly that $\hat{x}$ could follow $x$ after a short period of less than one second.

\section{Conclusion}

In this paper, we proposed a controller for a class of nonlinear systems with a clear-cut state observer. The main contribution in this paper is the design of the controller, which does not involve the system state directly, to forcing the output of the system to follow its desired value while maintaining all signals involved in the system stable. Also, it showed theoretically that the estimated state from the state observer follows its corresponding real state as well. Simulation results for a nonlinear system confirmed the theoretic analysis.

(Manuscript received April 22, 2004,

revised Nov. 1, 2004) 


\section{References}

(1) K. Tanaka and M. Sugeno: "Stability analysis and design of fuzzy control systems", Fuzzy Sets and Systems, Vol.45, pp.135-156 (1992)

( 2 ) L.-X. Wang: "Stable adaptive fuzzy control of nonlinear systems", IEEE trans. Fuzzy Systems, Vol.1, No.2, pp.146-155 (1993)

(3) C.-Y. Su and Y. Stepanenko: "Adaptive control of a class of nonlinear systems with fuzzy logic", IEEE trans. Fuzzy Systems, Vol.2, No.4, pp.285-294 (1994)

(4) M.M. Polycarpou: "Stable adaptive neural control scheme for nonlinear systems", IEEE Trans. on Automatic Control, Vol.41, No.3, pp.447-451 (1996)

(5) M.M. Polycarpou and M.J. Mears: "Stable adaptive tracking of uncertain systems using nonlinear parameterized on-line approximators", Int. J. Control, Vol.73, No.3, pp.363-384 (1998)

(6) H. Han, C.-Y. Su, and Y. Stepanenko: "Adaptive control of a class of nonlinear systems with nonlinearly parameterized fuzzy approximators", IEEE Trans. on Fuzzy Systems, Vol.9, No.2, pp.315-323 (2001)

( 7 ) N. Golea, A. Glolea, and K. Benmahammed: "Fuzzy model reference adaptive control", IEEE Trans. on Fuzzy Systems, Vol.10, No.4, pp.436-444 (2002)

(8) X.-J. Ma, Z.-Q. Sun, and Y.-Y. He: "Analysis and design of fuzzy controller and fuzzy observer", IEEE Trans. on Fuzzy Systems, Vol.6, No.1, pp.41-51 (1998)

( 9 ) C.-H. Wang, H.-L. Liu, and T.-C. Lin: "Direct adaptive fuzzyneural control with state observer and supervisory controller for unknown nonlinear dynamical systems", IEEE Trans. on Fuzzy Systems, Vol.10, No.1, pp.39-49 (2002)

(10) S. Tong and H.-X. Li: "Fuzzy adaptive sliding-mode control for MIMO nonlinear systems", IEEE Trans. on Fuzzy Systems, Vol.11, No.3, pp.354-360 (2003)

(11) J.-J. Slotine and J.A. Coetsee: "Adaptive sliding controller synthesis for nonlinear systems", Int. J. Control, Vol.43, pp.1631-1651 (1986)
(12) R. Palm: "Robust control by fuzzy sliding mode", Automatica, Vol.30, No.9, pp.1429-1437 (1994)

(13) H.K. Khalil: "Robust servomechanism output feedback controllers for feedback linearizable sytems", Automatica, Vol.30, No.10, pp.1587-1599 (1994)

(14) F. Esfandiari and Hassan K. Khalil: "Output feedback stabilization of fully linearizable systems", Int. J. Control, Vol.56, No.5, pp.1007-1037 (1992)

(15) H.K. Khalil: "Adaptive output feedback control of nonlinear systems represented by input-output models", IEEE Trans. on Automatic Control, Vol.41, No.2, pp.177-188 (1996)

(16) P.V. Kokotovic, H.K. Khalil, and J. O'Reilly: Singular Perturbation Methods in Control: Analysis and Design, Academic Press (1986)

(17) L.-X. Wang and J. M. Mendel: "Fuzzy basis functions, universal approximation, and orthogonal least-squares learning", IEEE Trans. on Neural Networks, Vol.3, pp.807-813 (1992)

Hugang Han (Member) Hugang Han received the B.E. de-

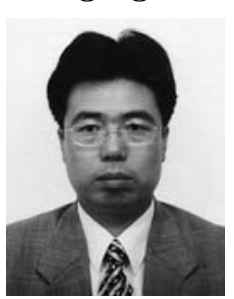
gree in control engineering from Northeast China Institute of Electric Power Engineering, Jilin, P.R.China in 1989, M.S. degree in mechanical engineering from Yamagata University in 1992, and Ph.D. degree in electric engineering from Kyushu Institute of Technology in 1997. From 1992 to 1994 he was with the Department of Automation at Northeast China Institute of Electric Power Engineering. Since 1997, he has been with Hiroshima Prefectural University where he is currently an Associate Professor of the Department of Management and Information Sciences.

Dr. Han's main research interests are in fuzzy control, adaptive control and information extraction. 